\title{
Molecular binding signatures of Trigonella foenum-graecum compounds on cyclin- dependent kinase 4 for possible anti-cancer mechanism in breast cancer
}

\author{
Enejoh Ojochenemi Aladi 1,2*, Rasheed-Jada Hadiza ${ }^{1}$, Eniafe Gabriel Opeyemi 2, Ogunleye Adewale Joseph ${ }^{2}$, \\ Oribamise Eunice Iyanuoluwa ${ }^{2}$, Adelakun Niyi Samuel ${ }^{2}$, Metibemu Samuel Damilohun ${ }^{2}$ and Omotuyi \\ Olaposi Idowu 2 \\ ${ }^{1}$ Genetics, Genomics \& Bioinformatics Department, National Biotechnology Development Agency (NABDA), FCT, Abuja, \\ Nigeria. \\ 2 Centre for Bio-Computing and Drug Development, Adekunle Ajasin University, Akungba-Akoko, Ondo State, Nigeria.
}

Publication history: Received on 25 May 2020; revised on 10 June 2020; accepted on 11 June 2020

Article DOI: https://doi.org/10.30574/gscbps.2020.11.3.0160

\begin{abstract}
Breast cancer (BC) continues to impose high epidemiological and socio-economic burden on female Homo sapiens, even in the face of new therapeutics. Of particular interest here is its neglected rise in low- and middle-income countries, thus necessitating the need for cost effective alternatives. The current understanding of a typical oncogenic BC cell dictates aberrant interplay, downstream the cyclin-dependent kinases (CDKs). CDKs play critical roles in the anomalous multiplicity of cancer cells and thus, represent interesting targets. In this report, structure-based virtual screening was carried out. The binding of compounds from Trigonella foenum-graecum (TF) to cyclin-dependent kinase 4 (CDK4) compared with that of standard drugs - Abemaciclib, Palbociclib and Ribociclib - was studied. In the light of palbociclib, a heuristic pipeline was designed to ensure specificity and selectivity of new inhibitors capable of antagonizing CDK4mediated phosphorylation of retinoblastoma protein $(\mathrm{Rb})$, a crucial step in abrogating neoplastic BC growth. The 2D conformations of phytocompounds were downloaded and optimised before being docked into the active site of CDK4 protein. In silico protocol to predict the drug-likeness of lead compounds, was also carried out. Five (5) phytocompounds emerged with binding energies lower than the standard drugs. Upon screening of lead compounds for their drug-likeness, four emerged as drug candidates: Irilone, Calycosin, Daidzein and Formononetin, which complied with Lipinski's "Rule of Five" (R05). Compounds from TF were found to exhibit lower binding energies, compared to standard drugs and could be potent in cancer treatment, especially human epidermal growth factor receptor 2 (HER2)-positive (+) BC.
\end{abstract}

Keywords: Trigonella foenum-graecum; breast cancer; CDK 4; HER2; palbociclib; in silico

\section{Introduction}

Cancer is a major public health disease which accounts for gross morbidity and mortality globally. BC, the leading female cancer accounts for $30 \%$ of new cancer cases and 14\% of deaths, and still remains a global burden [1]. Meanwhile, management and therapeutic options crucial for early diagnosis, therapy and counselling are lacking in low- and middleincome countries, thus causing gruesome social, economic, financial, and maternal health concerns.

Upon various scientific researches and further reviews, BC has been classified based on histopathological appearance, grade, stage, receptor status, amongst other classes. With respect to the receptor status, BC cells have receptors on their surface, nucleus or cytoplasm which may include estrogen receptor, progesterone receptor, or HER2. The present study will focus on HER2, which has been reported to be over expressed in about 25-30\% of BCs [2]. When stimulated by certain growth factors, HER2 causes cellular growth and division. BCs that test positive to this protein are thus referred

\footnotetext{
* Corresponding author: Enejoh Ojochenemi Aladi; e-mail: chenemidala@gmail.com
} 
to as HER2 + BCs, which when left untreated have shown to be more "aggressive" than other types of BCs [3]. Tumors with HER2 over expression and gene amplification tend to display biological aggressiveness and poor prognosis both in $\mathrm{BC}$ and other solid cancers [4,5]. Although the mechanism of selective amplification of $H E R 2 /$ neu is unknown, the HER2/neu oncogene in the amplicons on chromosome 17, when amplified, cause a marked increase in the HER2 expression on the surface of breast tumor cells. Following amplification, it is thought that the HER2 phenotype is fixed throughout the 'natural history' of the invasive tumor, which are manifest in HER2+ BCs [6].

It is important to note that alterations of the cyclinD/CDK/Rb pathway arise in about $90 \%$ of cancers [7]. Different molecular alterations, most frequently, cyclin D1 over expression, result in the disruption of the pathway in about 50$70 \%$ of BCs, particularly HER2 + BCs [8]. In HER2+ BC, cyclin D1 and CDK4 are critical drivers of cell proliferation [9] so, inhibiting CDK4 has represented a sound approach for abrogating neoplastic multiplicity.

Generally, CDKs are serine/threonine kinases which cooperate with their respective cyclins during the cell cycle. In cancer cell cycle, specifically during the gap1 phase, aberrant CDK4 cooperate with cyclin D to induce phosphorylation of $\mathrm{Rb}$. This stimulates the decoupling of E2F transcriptional factor and deactivation of the associated INK4 repressor, thereby ensuring a smooth and uncontrolled transition into the synthesis phase [10,11].

In light of this, CDK inhibitors (CDKIs) have been developed in the past decade as either monotherapy or combination therapy targeting CDK4. Their selectivity and specificity for CDK4 with admirable $\mathrm{IC}_{50}$ make them attractive options for BC therapy. CDKIs that have been developed for BC therapy include: Flavopiridol, Abemaciclib, Palbociclib and Ribociclib, however, toxicities and side effects have been recorded from the use of these drugs [12 - 16]. A generally insignificant survival rate, treatment and management option of BC still plague the human populace globally. This has necessitated the choice of plants as a potential alternative source of therapeutic intervention for prevention and treatment of BC. Some of these compounds are produced as secondary metabolites and are referred to as phytocompounds.

Natural products (from plants, microbes and animals) are said to be the single most productive source of leads for the development of drugs. These compounds on average, are more readily absorbed than synthetic drugs [17]. TF, commonly called Fenugreek, is a natural edible spice. A review of the pharmacological effects of the plant reveals it possesses hypoglycemic, anti-inflammatory and immunomodulatory activities. The seeds of the plant have been reported to be cytotoxic in vitro to a panel of cancer but not normal cells, the mechanism being through growth inhibition due to induction of cell death [18]. Diosgenin, a component of TF has been most studied of all the compounds found in this plant, and was reported to inhibit liver X receptor alpha activity in HepG2 cells and decreases plasma and hepatic triglycerides in obese diabetic mice as well as improve glucose metabolism by promoting adipocyte differentiation and inhibiting inflammation in adipose tissues [19].

However, little scientific information is available on other compounds present in TF and their therapeutic effects, especially their anti-cancer effects. This study therefore sets out to find selective inhibitor compounds present in TF, which may have physiologically relevant energetic consensus with CDK4.

\section{Computational methods}

\subsection{Protein molecule generation}

Crystal structure defining CDK4 (2W96) was retrieved from the Protein Data Bank (PDB: http://www.rcsb.org/pdb/). Already reported phytocompounds found in TF were mined from existing scientific literature. The 2D chemical conformers available on Pubchem compound database (https://www.ncbi.nlm.nih.gov/pccompound) were retrieved.

\subsection{Molecular docking}

The Schrodinger small molecule drug discovery suite, version 11.2.013; release 2017-2 on Linux-x86_64 platform was used for this virtual screening study. The 2D chemical conformers retrieved were optimized for docking using LigPrep tool. Optimization was performed with OPLS3 force field, possible ionization states generated at target $\mathrm{pH} 7.0 \pm 2.0$ using Epik. Energy minimization of CDK4 was carried out by protein preparation wizard, which involves the addition of missing hydrogen atoms, removal of unwanted water molecules, and assigning bond orders, partial charges and atom types. Ligand docking was carried out using standard precision (SP) and extra precision (XP) settings. Glide scores of the phytocompounds were obtained and compared to the standard drugs. 


\subsection{ADME/Tox screening}

The prediction of absorption, distribution, metabolism, excretion and toxicity (ADME/Tox) as well as drug-likeness of lead compounds were determined using Schrodinger QikProp tool. The Lipinski's R05 was applied to determine a lead compound's suitability to be developed into a drug [20,21].

\subsection{Structure analysis}

The 2D-ligand interaction diagrams were generated with Maestro 11.2 interface (Schrodinger).

\section{Results and discussion}

In this study the binding affinity and the chemical interactome of selected TF phytocompounds were probed via a heuristic in silico pipeline. The crucial nexus of CDK4 in furthering oncogenic cell proliferation makes it a sound target. Secondary metabolites produced by plants, commonly referred to as phytochemicals have been reported to be core determinants of the therapeutic use of plant parts. The drugs Palbociclib and Abemaciclib potently inhibit $\mathrm{Rb}$ phosphorylation, Ribociclib is however less potent. Abemaciclib, apart from inhibiting CDK4 and CDK6, has additional inhibitory activity for CDK9. Ribociclib inhibits both CDK4 and CDK6, but has the lowest binding scores when compared with the other two standards. Palbociclib will only inhibit CDK4 and CDK6 and has been reported to induce cell cycle arrest by haltering the phosphorylation of Rb leading to down regulation of E2F targeted genes [22, 23]. For this reason, it was chosen as a standard to further screen for compounds that could emerge as leads.

\subsection{Molecular docking and bonding interactions}

Target protein and phytocompounds used in this study, were prepared before proceeding to carry out docking protocols. The binding energy values (in $\mathrm{Kcal} / \mathrm{mol}$ ) of selected phytocompounds docked into the CDK4 active site compared to Palbociclib, a highly selective CDK4 inhibitor, are displayed in figure 1. Of all the compounds docked, Fenugreekine, Irilone, Calycosin, Daidzein, and Formononetin had lower binding scores (-10.47, -6.52, -6.37, -6.11 and $-5.63 \mathrm{Kcal} / \mathrm{mol}$, respectively) compared to the standards: Palbociclib, Abemaciclib, Ribociclib $(-5.55,-4.21,-3.76$ $\mathrm{Kcal} / \mathrm{mol}$, respectively). The low binding energies of the aforementioned phytocompounds may suggest that they have a greater affinity for the protein compared to standards. They were therefore selected as lead compounds and further computational analyses were carried out on them.


Figure 1 Glide scores of selected TF phytocompounds docked into the active site of CDK4

The physiological relevance of docking and docking interactions is a function of the ligand's ability to form bonds with the crucial amino acid residues that make up the catalytic triad. The hydrogen bond network and order of chemical reaction between CDK4, lead compounds and Palbociclib are shown in figure 2. Amino acids found in the binding pocket of CDK4 when bound to Palbociclib are associated with those found when bound to the phytocompounds. These residues are those found to be important in the ATP binding pocket of the kinase [24]. From figure 2, all the lead compounds form hydrogen bond with LYS35. This suggests that it might be an important residue for ligand binding. Palbociclib, Irilone and daidzein additionally form hydrogen bond with ARG101. Palbociclib and Formononetin also form hydrogen bond with LYS142 \& ALA16. Irilone and Daidzein form hydrogen bond with GLU94, while Calycosin and Formononetin interact with VAL96 via hydrogen bond formation. 
Enejoh et al. / GSC Biological and Pharmaceutical Sciences, 2020, 11(03), 014-021
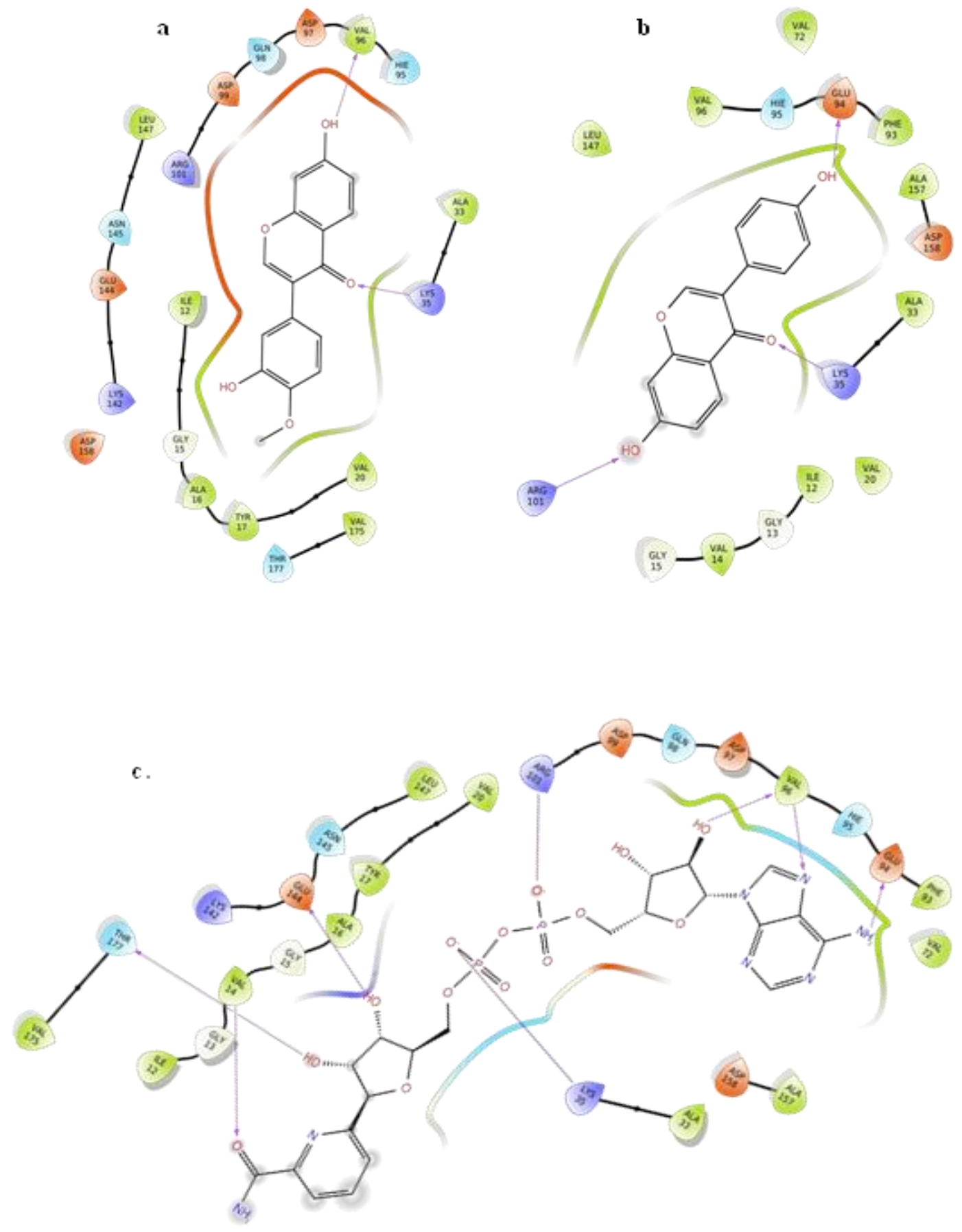

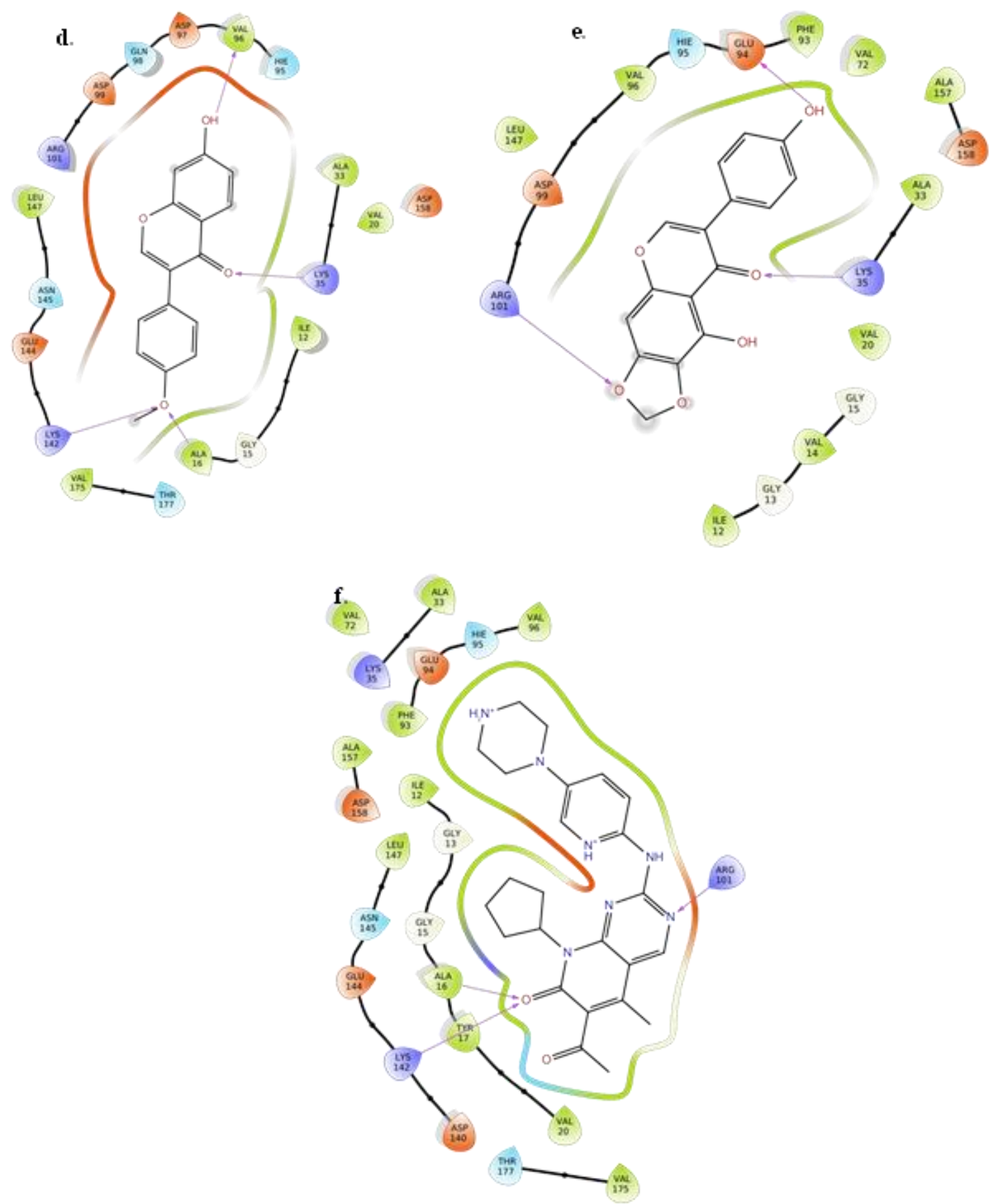

Figure 2 Ligand interaction diagrams of TF compounds (a-Calycosin; b-Daidzein; c-Fenugreekine; d-Formononetin; eIrilone) and Palbociclib (f) with CDK4

Hydrophobic interactions are said to be essential for protein stability [25]. Here, Palbociclib forms hydrophobic interactions in the CDK4 pocket with the amino acid residues ILE12, ALA33, VAL72, PHE93 \& ALA157. in addition to the aforementioned residues, Irilone makes hydrophobic contact with ASP99, which may also contribute to its low binding energy. Hydrophobic contact between Calycosin and ALA16, TYR17, VAL20 \& VAL175 residues were observed. DaidzeinCDK4 complex may have been stabilized by forming hydrophobic bond formation with ILE12, VAL20, PHE93, VAL96, LEU147 \& ALA157 residues. Formononetin as well, interacts with CDK4 residues - ILE12, GLY15, ALA16, VAL20 \& ALA33 via hydrophobic bonds. 


\subsection{Validation of molecular docking results}

Molecular docking employed in this study was validated by docking already established antagonist compounds obtained from the available online ChEMBL database (https://www.ebi.ac.uk/chembl/). The FASTA sequence of the 3D crystal structure of CDK4 was copied from the PDB and blasted on the ChEMBL website. CDK4 (CHEMBL331) was chosen. The compounds obtained from these experiments were docked into the same ligand-binding pocked used for the phytocompounds. A correlation coefficient graph between the glide scores of the compounds generated from docking and their corresponding ChEMBL's Pubchem values (experimentally determined IC50) was plotted. The graph (figure 3) revealed a strong correlation between the docked scores and the experimentally derived data $(r=0.74)$; this gives credence to the fact that computational docking can replicate experimental data. It also shows that the docking carried out in this study, is dependable.

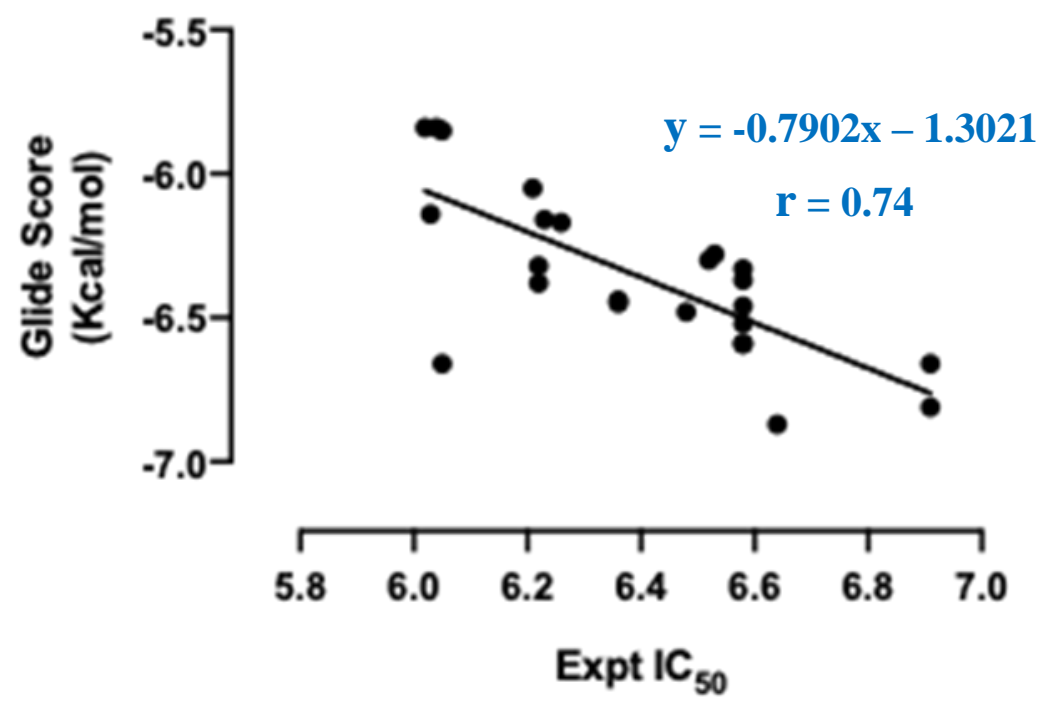

Figure 3 Correlation plot of $\mathrm{pIC}_{50}$ (experimental, $\mathrm{CHEMBL}$ ) versus glide score

\subsection{ADME/Tox screening}

The process of developing a lead compound into a drug is tedious and requires enormous resources to be put in place. At the end of the day, too many drug candidates fail as a result of unsatisfactory ADME/Tox properties. ADME/Tox provide information on the pharmacokinetic properties of a compound, which aids in determining the suitability of lead compounds in the drug discovery pipeline [20,21]. The Lipinski's R05 expresses molecular properties significant for evaluating drug-likeness of compounds. The rule states that any orally active drug should obey these criteria: it must have a molecular weight $<500$ daltons, hydrogen bond donors $\leq 5$, hydrogen bond acceptors $\leq 10$ and octanol-water partition coefficient $(\log \mathrm{Po} / \mathrm{w})<5$ [20]. A compound which violates three $(3)$ or more of the rule, is regarded unsuitable for further drug development. The drug-likeness of the standards and lead compounds are summarised in table 1. Palbociclib, like its other counterparts - Abemaciclib and Ribociclib, meet the criteria for a drug. Fenugreekine on the other hand, violated 3 of the rules, and even though it exhibited the lowest binding to the protein, is not fit to proceed as a drug candidate as it could exhibit some toxic properties. The others however obeyed the rules without any violations and so could be suitable as drug candidates. 
Table 1 ADME/Tox properties of compounds

\begin{tabular}{llllll}
\hline Compound & Mol Wt. (daltons) & HBD & HBA & QPlogPo/w & RO5 violation \\
\hline Palbociclib & 447.54 & 2 & 11 & 1.991 & 0 \\
Abemaciclib & 506.60 & 1 & 9 & 4.556 & 1 \\
Ribociclib & 434.54 & 2 & 9 & 3.184 & 0 \\
Fenugreekine & 663.43 & 8 & 25.7 & -3.186 & 3 \\
Irilone & 298.25 & 1 & 4.5 & 1.96 & 0 \\
Calycosin & 284.27 & 2 & 4.75 & 1.901 & 0 \\
Daidzein & 254.24 & 2 & 4 & 1.728 & 0 \\
Formononetin & 268.27 & 1 & 4 & 2.586 & 0 \\
\hline
\end{tabular}

Mol Wt. - Molecular weight; HBD - Hydrogen bond donor; HBA - Hydrogen bond acceptor; QPlogPo/w - Octanol-water partition coefficient; R05 Rule of five.

\section{Conclusion}

This study investigated the binding of compounds present in TF to CDK4. Previous reports indicate that TF possesses anti-cancer effects, however, the compounds responsible and the mechanism through which they act have not been reported. Data obtained from this study suggests TF compounds could be potent in cancer treatment, especially HER2 $+\mathrm{BC}$, given that they can inhibit CDK4 which represents a critical transition factor of a cell from the gap 1 phase to the synthesis phase during cell proliferation. To further validate this claim, in vitro and in vivo assays are recommended.

\section{Compliance with ethical standards}

\section{Disclosure of conflict of interest}

Authors declare no conflict of interests.

\section{References}

[1] Siegel RL, Miller KD and Jemal A. (2017). Cancer Statistics, 2017. CA: A Cancer Journal for Clinicians, 67(1), 7-30.

[2] Mitri Z, Constantine T and O'Regan R. (2012). The HER2 receptor in breast cancer: pathophysiology, clinical use, and new advances in therapy. Chemotherapy research and practice.

[3] Sotiriou C and Pusztai L. (2009). Gene-expression signatures in breast cancer. New England Journal of Medicine, 360(8), $790-800$.

[4] English DP, Roque DM and Santin AD. (2013). HER2 expression beyond breast cancer: therapeutic implications for gynecologic malignancies. Molecular diagnosis \& therapy, 17(2), 85 - 99.

[5] Yan M, Parker BA, Schwab R and Kurzrock R. (2014). HER2 aberrations in cancer: implications for therapy. Cancer treatment reviews, 40(6), 770 - 80.

[6] Burstein HJ. (2005). The distinctive nature of HER2-positive breast cancers. New England Journal of Medicine, 353(16), 1652.

[7] Altenburg JD and Farag SS. (2015). The potential role of PD0332991 (Palbociclib) in the treatment of multiple myeloma. Expert opinion on investigational drugs, 24(2), $261-71$.

[8] Peurala E, Koivunen P, Haapasaari KM, Bloigu R and Jukkola-Vuorinen A. (2013). The prognostic significance and value of cyclin D1, CDK4 and p16 in human breast cancer. Breast Cancer Research, 15(1), R5. 
[9] Choi YJ, Li X, Hydbring P, Sanda T, Stefano J, Christie AL, Signoretti S, Look AT, Kung AL, von Boehmer H and Sicinski P. (2012). The requirement for cyclin D function in tumor maintenance. Cancer cell, 22 (4), 438 - 51.

[10] Baker SJ and Reddy EP. (2012). CDK4: a key player in the cell cycle, development, and cancer. Genes \& cancer, 3(11-12), $658-69$.

[11] Shapiro GI. (2006). Cyclin-dependent kinase pathways as targets for cancer treatment. Journal of Clinical Oncology, 24(11), 1770 - 1783.

[12] Hamilton E and Infante JR. (2016). Targeting CDK4/6 in patients with cancer. Cancer treatment reviews, 45, 129 -38 .

[13] Xu H, Yu S, Liu Q, Yuan X, Mani S, Pestell RG and Wu K. (2017). Recent advances of highly selective CDK4/6 inhibitors in breast cancer. Journal of hematology and oncology, 10(1), 97.

[14] Finn RS, Aleshin A and Slamon DJ. (2016). Targeting the cyclin-dependent kinases (CDK) 4/6 in estrogen receptor-positive breast cancers. Breast Cancer Research, 18(1), 17.

[15] Sherr CJ, Beach D and Shapiro GI. Targeting CDK4 and CDK6: from discovery to therapy. Cancer Discovery, 6 (4), $353-367$.

[16] Vidula N and Rugo HS. (2016). Cyclin-dependent kinase 4/6 inhibitors for the treatment of breast cancer: a review of preclinical and clinical data. Clinical breast cancer, 16(1), 8 - 17.

[17] Harvey AL. (2008). Natural products in drug discovery. Drug discovery today, 13(19-20), $894-901$.

[18] Shabbeer S, Sobolewski M, Anchoori RK, Kachhap S, Hidalgo M, Jimeno A, Davidson NE, Carducci M and Khan SR. (2009). Fenugreek: a naturally occurring edible spice as an anticancer agent. Cancer biology and therapy, 8(3), 272 - 278.

[19] Uemura T, Hirai S, Mizoguchi N, Goto T, Lee JY, Taketani K, Nakano Y, Shono J, Hoshino S, Tsuge N and Narukami T. (2010). Diosgenin present in fenugreek improves glucose metabolism by promoting adipocyte differentiation and inhibiting inflammation in adipose tissues. Molecular nutrition and food research, 54(11), 1596 - 608.

[20] Lipinski CA. (2004). Lead-and drug-like compounds: the rule-of-five revolution. Drug Discovery Today: Technologies, 1(4), $337-41$.

[21] Lipinski CA, Lombardo F, Dominy BW and Feeney PJ. (1997). Experimental and computational approaches to estimate solubility and permeability in drug discovery and development settings. Advanced drug delivery reviews, 23(1-3), 3 - 25.

[22] Chen P, Lee NV, Hu W, Xu M, Ferre RA, Lam H, Bergqvist S, Solowiej J, Diehl W, He YA and Yu X. (2016). Spectrum and Degree of CDK drug interactions predicts clinical performance. Molecular cancer therapeutics, 15(10), 2273 -2281 .

[23] Lu J. (2015). Palbociclib: a first-in-class CDK4/CDK6 inhibitor for the treatment of hormone-receptor positive advanced breast cancer. Journal of hematology and oncology, 8(1), 98.

[24] Rondla R, PadmaRao LS, Ramatenki V, Haredi-Abdel-Monsef A, Potlapally SR and Vuruputuri U. (2017). Selective ATP competitive leads of CDK4: Discovery by 3D-QSAR pharmacophore mapping and molecular docking approach. Computational biology and chemistry, 71, 224- 229.

[25] Pace CN, Fu H, Fryar KL, Landua J, Trevino SR, Shirley BA, Hendricks MM, Iimura S, Gajiwala K, Scholtz JM and Grimsley GR. (2011). Contribution of hydrophobic interactions to protein stability. Journal of molecular biology, 408(3), $514-528$.

\section{How to cite this article}

Enejoh OA, Rasheed-Jada H, Eniafe GO, Ogunleye AJ, Oribamise EI, Adelakun NS, Metibemu SD and Omotuyi OI. (2020). Molecular binding signatures of Trigonella foenum-graecum compounds on cyclin-dependent kinase 4 for possible anticancer mechanism in breast cancer. GSC Biological and Pharmaceutical Sciences, 11(3), 14-21. 